\title{
Impacto social y económico del capital humano en las microempresas: un contraste colombiano y global
}

\author{
DEIVI DAVID FUENTES DORIA* \\ MELISA ANDREA FAJARDO PEREIRA** \\ JOSÉ LUIS DÍAZ BALLESTEROS*** \\ JOHANA ELISA FAJARDO PEREIRA****
}

\footnotetext{
* Postdoctorado en Gestión de la Ciencia y la Tecnología. Universidad Pontificia Bolivariana, Montería, Colombia. E-mail: deivi.fuented@upb.edu.co. ORCID: 0000-0oo2-0699-286X. Google Scholar: https://scholar.google.es/ citations?user=NfH3bgEAAAAJ\&hl=es. Scopus Author ID: https://www.scopus.com/authid/detail.uri?authorld=57211404164.

** Doctoranda en Ciencias-Gerencia. Universidad Rafael Belloso Chacín, Maracaibo, Venezuela. E-mail: mfajardo@fca.edu.co. ORCID: 0000-0001-6940-8723. Google Scholar: https://scholar.google.es/citations?hl=es\&user=xTQGfloAAAAJ.

*** Magíster en Administración e Innovación. Universidad Pontificia Bolivariana, Montería, Colombia. E-mail: jose.diazb@upb.edu.co. ORCID: 0000-0002-8296-7156. Google Scholar: https://scholar.google.com/citations?user=Cx21Vp8AAAAJ\&h=es.

**** Doctoranda en Ciencias-Gerencia. Universidad Cooperativa de Colombia, Montería, Colombia.E-mail: johanafajardo@hotmail.com. ORCID: 0000-0001-7963-9349. Google Scholar: https://scholar.google.es/citations?hl=es\&user=YZklqQQAAAAJ.
} 


\section{COMO CITAR ESTE ARTÍCULO}

How to cite this article:

Fuentes, D. et al. (2021). Impacto social y económico del capital humano en las microempresas: un contraste colombiano y global. Revista Perspectiva Empresarial, 8(2), 104-121.

Recibido: 01 de junio de 2021 Aceptado: 08 de octubre de 2021
RESUMEN El capital humano es un factor determinante en las empresas, aunque la crisis económica y social que vive el mundo a causa del brote del coronavirus le ha producido afectaciones de gran impacto debido a las altas tasas de desempleo. Objetivo. Describir las afectaciones causadas al capital humano en las microempresas durante la emergencia social y económica a causa de la COVID-19. Metodología. La metodología se basa en una revisión de publicaciones en la base de datos Scopus con el uso del software VOSviewery un contraste con los reportes generados por los órganos de control en Colombia. Resultados. Los resultados muestran las principales problemáticas que vive el capital humano a nivel global como son altas tasas de desempleo que aumentaron los suicidios, la recesión económica de las empresas, la afectación en la salud mental de los trabajadores y los efectos económicos por el cese de actividades. Conclusiones. Se concluye que las afectaciones a la pérdida de empleos estuvieron más concentradas en algunas actividades como el transporte de pasajeros y los servicios de turismo.

PALABRAS CLAVE capital humano, COVID-19, emergencia social y económica, MiPymes.

\section{Social and economic impact of human capital in micro-enterprises: A Colombian and global contrast}

ABSTRACT Human capital is a determining factor in companies; however, the global economic and social crisis caused by the coronavirus outbreak has had a significant impact due to high unemployment rates. Objective. To describe the effects of COVID-19 on human capital in microenterprises during the social and economic emergency. Methodology. The methodology is based on a review of publications in the Scopus database using the VOSviewer software and a contrast with reports generated by control bodies in Colombia. Results. The findings reveal the major issues confronting human capital on a global scale, such as the high unemployment rates that increased suicide rates, firms' economic recessions, worker mental health issues, and the economic consequences of the cessation of activities. Conclusions. It is determined that the effects of job losses were highest in certain activities such as passenger transport and tourism services.

KEY WORDS Human capital, COVID-19, social and economic emergency, MSMEs. 


\section{Impacto social e económico do capital humano nas microempresas: um contraste colombiano e global}

RESUMO O capital humano é um fator determinante nas empresas, embora a crise econômica e social que o mundo atravessa devido ao surto do coronavírus tenha tido um grande impacto sobre ele devido às altas taxas de desemprego. Objetivo. Descrever os efeitos sobre o capital humano nas microempresas durante a emergência social e econômica causada pela COVID-19. Metodologia. A metodologia é baseada em uma revisão de publicações na base de dados Scopus com o uso do software VOSviewer e um contraste com os relatórios gerados pelos órgãos de controle na Colômbia. Resultados. Os resultados mostram os principais problemas experimentados pelo capital humano a nível global, como as elevadas taxas de desemprego que aumentaram os suicídios, a recessão econômica das empresas, a afetação à saúde mental dos trabalhadores e os efeitos econômicos decorrentes da cessação das atividades. Conclusões. Conclui-se que os efeitos da perda de empregos foram mais concentrados em algumas atividades, como o transporte de passageiros e os serviços de turismo.

PALAVRAS CHAVE capital humano, COVID-19, emergência social e econômica, MPMEs. 


\section{Introducción}

El mundo ha sufrido una de las crisis sociales más fuertes en los últimos 100 años por consecuencia del brote del coronavirus (SARSCoV-2), el cual ha cobrado miles de vidas en todos los países, causando un desequilibrio en las economías globales (Korsgaard etal., 2020; Nwosu and Oyenubi, 2021). Los gobiernos con el fin de contrarrestar los brotes y contagios han adoptado medidas de aislamiento obligatorios preventivos y prohibiciones de circulación en todo el territorio (Yarovaya et al., 2021). Estas medidas han sido favorables para disminuir las curvas de contagio pero también han causado un efecto negativo en las empresas, en especial en las micro, pequeñas y medianas empresas - MiPymes-(Lu etal., 2020).

Algunos de estos efectos plasmados en estudios recientes sobre las MiPymes mencionan como la COVID-19 afecta la economía, centrándose en los siguientes aspectos: la escasez de recursos de capital y el aumento de precios en los alimentos (Ahn and Norwood, 2021; Eggers, 2020; Lu et al., 2020; Villalobos et al., 2021); la eficiencia operativa del capital humano en relación con las actividades de los sectores financieros (Yarovaya et al., 2021; Mirza et al., 2020); la captación de capital o recursos económicos como fuente principal para el desarrollo de servicios o producción de bienes (Song, Yang and Tao, 2020); el acceso a líneas de crédito y financiación por parte de proveedores crediticios para la sostenibilidad de las empresas (Zimon and Dankiewicz, 2020); el riesgo de la COVID-19 en los factores de salud, educación y el transporte de personas (Amuda, 2020; Wahyono et al., 2021; Schmitz et al., 2021; Davila et al., 2021); las afectaciones por las medidas de distanciamiento social para evitar el aumento de contagios (Nande et al., 2021) y las altas tasas de desempleo (Nga, Ramlan and Naim, 2021; Nwosimiri, 2021; Svabova et al., 2021).

Todo este escenario de problemas alrededor de las MiPymes, causado por la crisis económica y social, ha ocasionado cierres de actividades económicas en todos los sectores. El último reporte generado por la Comisión Económica para América Latina y el Caribe -CEPAL- establece que los sectores productivos estiman 2,7 millones de empresas, la mayor parte microempresas, lo que implicaría una cifra de 8,5 millones de desempleados en todo el mundo. Esto, sin contar los empleos informales afectados por causa de las políticas de aislamiento que imposibilitan la circulación y el desempeño de su economía.

Ahora bien, como se ha especificado en diversos estudios mundiales, es difícil prever la intensidad y la duración que tendrá la crisis económica y social en cada territorio y sector puesto que cada país la vive de acuerdo a sus condiciones políticas y económicas (Islam, 2020; Pinilla et al., 2021). Sin embargo hay sectores con afectaciones clasificadas como fuertes, significativas y moderadas, según el grado de afectación. En primer lugar se encuentran los sectores de servicios de turismo, industrias culturales, comercio, reparación, hoteles, transporte, moda y automóviles (Burhan et al., 2021; Jílková, 2021; Konečný, Brídziková and Senko, 2021). En segundo lugar se distinguen los sectores de la minería, electricidad, construcciones, servicios empresariales, actividades financieras, bebidas y muebles. Finalmente en la categoría moderada por su bajo impacto- se encuentra la agricultura, producción de alimentos, insumos, medicamentos, telecomunicaciones y envases (CEPAL, 2020).

Debido a la diversidad en la intensidad por sectores surge una de las problemáticas más impactantes en la sociedad, la cual involucra el desempleo y por consiguiente a la calidad de vida de las personas. Para la CEPAL se proyectan 8,5 millones de desempleados; a esto se suma las actividades informales que no han podido retomar operación por falta de recursos, capital humano y obligaciones. Es así que una de las variables que genera la emergencia social y que afecta a la mayor parte de la población es la pérdida del empleo y la posibilidad de suplir las necesidades básicas de alimentación, educación y salud (Kim et al., 2021; Bikse et al., 2021; Restrepo, Rabbitt and Gregory, 2021).

A partir de lo descrito, este estudio pretende mostrar los efectos que ha dejado el brote del coronavirus en el capital humano en las empresas MiPymes. La primera parte del texto se enfoca en revisar la evolución de estudios asociados al capital humano y los efectos de la COVID-19. La segunda parte analiza las principales problemáticas del capital humano por países y en la tercera se realiza un contraste con las realidades del contexto colombiano. 


\section{Riesgos empresariales de las MiPymes a nivel mundial}

La economía sufrió una pérdida notable cuando la COVID-19 golpeó los negocios y los llevó al cierre. Las repercusiones de la caída económica debido al cierre de negocios a causa de las políticas de distanciamiento social y del confinamiento han hecho que las pequeñas empresas pierdan los mecanismos de operación y vean una disminución de las ganancias, afectando también a más de 22 millones de personas que solicitan empleo (Kyung and Whitney, 2020).

Es más que claro, que la crisis social y económica es fuerte en todo el mundo. Aun en el 2021 las condiciones económicas son desfavorables para las MiPymes, las cuales ocupan más del $90 \%$ del tejido empresarial de la economía mundial (Rodríguez, Díaz y Niebles, 2018). Por tanto, estudiar los efectos económicos y financieros de este sector ofrece una visión general de la condición empresarial de los países.

De este modo es preciso mencionar que la emergencia de la COVID-19 ha cambiado el comportamiento de las personas y la forma de consumo a la hora de comprar. Se puede apreciar que una mayor población opta por las compras en línea para evitar contagios y aglomeraciones en las tiendas y centros de comercio. Situación que es muy desfavorable para las MiPymes porque no estaban preparadas para comercializar sus productos por estos mecanismos tecnológicos, aunque algunas han logrado migrar a plataformas digitales para sostenerse en el mercado (Soelasih and Jaya, 2020).

Otro aspecto que ha afectado económica y socialmente a las MiPymes se asocia a los costos fijos y pagos de impuestos. Estos dos elementos han sido un factor de impacto en estas empresas. El primero se asocia a las condiciones de costos fijos que involucra gastos representativos tales como la nómina, arrendamientos y servicios. Si bien no existen estudios actuales que aborden este efecto, la realidad empresarial de las MiPymes es que han estado sumergidas en estos rubros tanto que incluso han sido tema de discusión por los gobiernos nacionales.
En los inicios de la crisis, muchos países adoptaron como medidas el cierre de actividades o la imposibilidad de realizar la actividad económica pero con indicaciones de continuar causando los gastos de nómina y de arriendos para acceder a ciertos beneficios; es así como algunos gobiernos brindaron estímulos monetarios de apoyo a su actividad empresarial; aunque el sector comercial, en especial, no cumplía con las condiciones establecidas por estos para acceder a dichas ayudas. Por su parte, las tasas impositivas de impuestos se mantienen a pesar de la pandemia; a la vez muchos gobiernos no implementaron estrategias transitorias para disminuirlos en busca de incentivar la economía y mejorar la situación financiera de las empresas (Makin and Layton, 2021); por tanto, todo esto ahondó la crisis.

\section{Metodología}

El estudio se centra en un análisis del impacto del capital humano y las afectaciones a partir de la emergencia social y económica causadas por la COVID-19. Para la obtención de información se consideraron dos momentos: en el primero se usó la base de datos Scopus, reconocida como una de las que reporta mayores estudios de relevancia (Fuentes-Doria, Toscano-Hernándezand Sepulveda, 2019). Se usaron los términos "unemployment", "human capital" en las "firm", "business", "company" y "SMEs", quedando la ecuación de búsqueda (TITLE-ABS-KEY (unemployment OR "human capital”) AND TITLE-ABS-KEY (COVID-19) AND TITLE-ABS-KEY (firm OR business OR company OR SMEs)); limitada a textos en inglés y español, con un resultante de 55 documentos. En el segundo momento se recopiló información de la Cámara de Comercio de Bogotá, obtenida de la "Encuesta nacional sobre el impacto económico COVID-19 Capítulo Bogotá".

Una vez descargado el documento de la base de datos a través de archivo plano se hizo el análisis a partir de dos contextos. Primero una revisión de aspectos relevantes y aportes, plasmado en artículos y capítulos de libros por medio de una matriz Excel en la que se organizaron aspectos relevantes de problemáticas y estrategias de las empresas en medio de la emergencia. En la segunda 
parte se hace un análisis sistemático con el uso del software VOSviewer, en este se pueden visualizar mapas bibliométricos de relación de los estudios y países que trabajan de manera colaborativa (Toscano-Hernández, Fuentes-Doria y FajardoPereira, 2019; Toscano-Hernández et al., 2020; Fuentes-Doria et al., 2020). Finalmente se hace un contraste de las problemáticas con el escenario de la región de Colombia.

\section{Resultados y discusión}

El analizar documentos científicos puede mostrar características interesantes para la comunidad académica y profesional. Lograr entender las problemáticas mundiales alrededor de un hecho o evento brinda a los interesados en la ciencia estructuras para desarrollar análisis comparativos que suelen aportar datos para la solución de problemáticas actuales. De acuerdo con esta disposición, este apartado pretende abordar aspectos descriptivos de los estudios alrededor del capital humano en medio de la emergencia social y económica de la COVID-19.

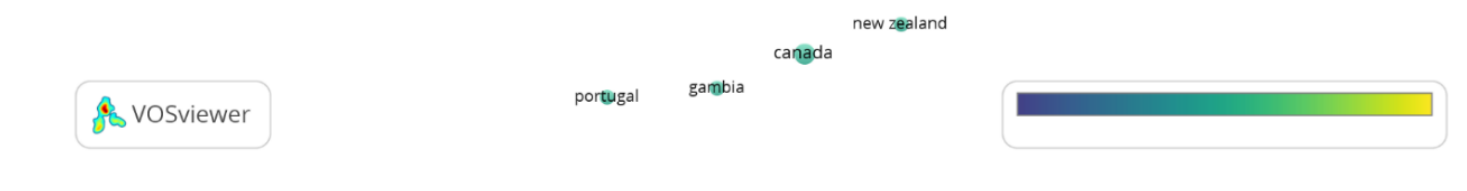

Figura 1. Redes de países con estudios alrededor de las MiPymes y capital humano. Fuente: elaboración propia por parte de los autores.

En este primer resultado de redes de países sobre estudios del capital humano en las MiPymes en medio de la emergencia social y económica se evidencian los trabajos colaborativos, lo que permite su clasificación en clúster. Los clústeres son grupos de países que trabajan de manera colaborativa para obtener un objetivo común a través de centros de investigación o instituciones universitarias. El primer clúster agrupa un número significativo de participantes en los que se encuentran Brasil, India, Libia, Suecia y Reino unido. En el segundo grupo están China, Hong Kong y Macao. En el tercer clúster se ubican Australia, Arabia Saudita y Bangladesh. El cuarto grupo está formado por Estados Unidos y República Dominicana, también Alemania e Italia. Los demás países que han realizado estudios son Gambia, Grecia, Indonesia, Kuwait, Malasia, Nueva Zelanda, Portugal, Sudáfrica, Macedonia del Norte y Suiza. 
Tabla 1. Estudios destacados del capital humano en medio de la emergencia social y económica de la COVID-19

\begin{tabular}{lll}
\hline \multicolumn{1}{c}{ Autores } & \multicolumn{1}{c}{ Titulo } & \multicolumn{1}{c}{ Citación } \\
\hline $\begin{array}{l}\text { lacus, S.M., Natale, F., Santamaria, } \\
\text { C., Spyratos, S., Vespe, M. }\end{array}$ & $\begin{array}{l}\text { Estimating and projecting air passenger traffic during the COVID-19 } \\
\text { coronavirus outbreak and its socio-economic impact }\end{array}$ & 23 \\
\hline $\begin{array}{l}\text { Bartik, A.W., Bertrand, M., Cullen, Z., } \\
\text { Glaeser, E.L., Luca, M., Stanton, C. }\end{array}$ & The impact of COVID-19 on small business outcomes and expectations & 16 \\
\hline $\begin{array}{l}\text { Shammi, M., Bodrud-Doza, M., Islam, } \\
\text { A.R.M.T., Rahman, M.M. }\end{array}$ & $\begin{array}{l}\text { Strategic assessment of COVID-19 pandemic in Bangladesh: comparative } \\
\text { lockdown scenario analysis, public perception, and management for } \\
\text { sustainability }\end{array}$ & $\begin{array}{l}\text { Labor issues and COVID-19 } \\
\text { Larue, B. }\end{array}$ \\
\hline $\begin{array}{l}\text { Mamun, M.A., Bhuiyan, A.K.M.I., } \\
\text { Manzar, M.D. }\end{array}$ & \begin{tabular}{l} 
The first COVID-19 infection are the causative factors \\
\hline
\end{tabular} & $\begin{array}{l}\text { Nigerian budgetary response to the COVID-19 pandemic and its shrinking } \\
\text { fiscal space: Financial sustainability, employment, social inequality and } \\
\text { business implications }\end{array}$ \\
\hline
\end{tabular}

Fuente: elaboración propia por parte de los autores.

La tabla 1 presenta los estudios más destacados en la temática de capital humano. Estos se seleccionaron por el factor de citación individual del sector tal como se describe en el texto denominado "Estimating and projecting air passenger traffic during the COVID-19 coronavirus outbreak and its socio-economic impact"; este se centra en analizar el impacto que ha tenido la prohibición de viajar, principalmente se habla de una reducción para el primer trimestre de 2020 del 0,02 \% y 0,12\% del PIB de cada país. Teniendo en cuenta otros efectos como son la pérdida de empleo, alrededor de un 4,2 y 5 millones a causa de los efectos del sector de aviación (Iacus et al., 2020).

De igual manera el texto que ha logrado un total de 16 citaciones denominado "The impact of COVID-19 on small business outcomes and expectations", el cual se centra en revisar todos los efectos económicos y sociales que han tenido las MiPymes a raíz de la COVID-19; las 5800 empresas analizadas muestran que surgieron despidos masivos a solo una semana del cierre de los establecimientos. Igualmente los cierres se asociaron a las inesperadas restricciones de distanciamiento social que establecieron los países y distintas regiones. Otro factor que se vinculó al cierre fue la incapacidad financiera de muchas de las MiPymes dado que son órganos frágiles respecto al capital, ya que su flujo logra cubrir gastos sin operaciones por períodos de máximo dos semanas (Bartik et al., 2020).

Por otro lado, el texto "Strategic assessment of COVID-19 pandemic in Bangladesh: comparative lockdown scenario analysis, public perception, and management for sustainability" se centró en revisar las percepciones públicas en los escenarios de bloqueo comparativo que pueden afectar los objetivos de sostenibilidad y régimen de estrategia en la pandemia. La investigación inicia revisando los efectos de los aportes públicos a los diferentes problemas sociales tales como tasa de muerte e infección, movimiento de personas en las comunidades, subida de precios por el comercio, violencia de género, educación formal y finaliza con la relación entre las personas con vida y la tasa de desempleo aumentada debido al cierre de muchos establecimientos comerciales (Shammi et al., 2020; Grashuis, 2021; Palma, Araos and Moore, 2021).

Del mismo modo el texto "Labor issues and COVID-19" se centra en revisar los efectos que ha tenido la enfermedad en las tasas de desempleo en los países, especialmente en Canadá. Los datos plantean que unas 500000 personas solicitaron 
subsidios de desempleo, situación similar que viven países como Estados Unidos donde 6,6 millones de personas se encuentran tramitando reclamos de subsidios laborales. La gravedad de la situación es consecuencia de la pandemia y la incertidumbre aumenta al no saber cuándo y cómo se levantarán las restricciones económicas (Larue, 2020).

A la vez el artículo "The first COVID-19 infanticide-suicide case: Financial crisis and fear of COVID-19 infection are the causative factors" hace un estudio de los factores de suicidios que se han reportado en la pandemia de la COVID-19 y en el que se encontró que la pérdida de los empleos y disminución de la capacidad económica en los hogares es uno de los más recurrentes. Se han destacado algunas evidencias empíricas de personas que han perdido su empleo y recurrieron al suicidio y a asesinatos de otros miembros del hogar, especialmente niños (Mamun, Bhuiyan and Manzar, 2020; David et al., 2021).

Finalmente el texto "Nigerian budgetary response to the COVID-19 pandemic and its shrinking fiscal space: Financial sustainability, employment, social inequality and business implications" se encarga de explorar las respuestas presupuestarias del gobierno nigeriano a partir de la COVID-19 y sus implicaciones económicas y sociales. El artículo explora los documentos presupuestarios de los gobiernos federales y las políticas circulares del Banco Central del país. Entre las evidencias se encuentra el aumento de préstamos para financiación de la emergencia social y entre los cuales las MiPymes han tenido intervención positiva (Ejiogu, Okechukwu and Ejiogu, 2020).

Tabla 2. Principales problemáticas del capital humano a partir de la COVID-19

\begin{tabular}{|c|c|c|}
\hline Países & Principales problemáticas & Estrategias \\
\hline Bangladesh & $\begin{array}{l}\text { Suicidios de familia a causa de la crisis } \\
\text { económica, desempleo. Limitación del sector } \\
\text { ganadero para comercialización de productos. }\end{array}$ & $\begin{array}{l}\text { Estrategias de promoción de salud mental para disminuir la } \\
\text { incidencia de casos de infanticidio-suicidio, subsidios de } \\
\text { desempleo para disminuir las tasas de suicidios. }\end{array}$ \\
\hline Sudáfrica & $\begin{array}{l}\text { Afectaciones de las actividades de ocio y turismo } \\
\text { por las políticas de distanciamientos social. }\end{array}$ & $\begin{array}{l}\text { Se adoptó la tecnología para conectarse con los demás y } \\
\text { realizar actividades de ocio. El futuro de las prácticas de } \\
\text { ocio y recreación posteriores a la COVID-19 no están claras } \\
\text { y cambiarán indefinidamente. }\end{array}$ \\
\hline Brasil & $\begin{array}{l}\text { Recesión económica y afectaciones en la atención } \\
\text { de salud. }\end{array}$ & $\begin{array}{l}\text { Inversión de recursos para mejorar las condiciones } \\
\text { económicas y sociales de los trabajadores de los centros } \\
\text { de salud. }\end{array}$ \\
\hline Reino Unido & $\begin{array}{l}\text { Cierres de establecimientos de comercio por las } \\
\text { políticas de distanciamiento social, lo que afecta } \\
\text { los niveles de venta. }\end{array}$ & $\begin{array}{l}\text { Proponer espacios dinámicos para el comercio y que brinden } \\
\text { seguridad a los consumidores. }\end{array}$ \\
\hline $\begin{array}{l}\text { Estados } \\
\text { Unidos }\end{array}$ & $\begin{array}{l}\text { Crisis mentales a partir de la crisis económica y } \\
\text { social, lo que involucra el riesgo de enfermedad } \\
\text { y la pérdida de empleo. }\end{array}$ & $\begin{array}{l}\text { Programas de atención mental y subsidios de empleo a la } \\
\text { población afectada. }\end{array}$ \\
\hline Rusia & $\begin{array}{l}\text { Políticas de presupuesto para atender la crisis } \\
\text { económica y social. }\end{array}$ & $\begin{array}{l}\text { Ajustes de políticas fiscales para las MiPymes para incentivar } \\
\text { los procesos económicos. Mantener los niveles de empleo } \\
\text { y capacidad monetaria del país. }\end{array}$ \\
\hline Australia & $\begin{array}{l}\text { Malas políticas fiscales sin beneficios a la } \\
\text { población afectada. }\end{array}$ & $\begin{array}{l}\text { Implementación de políticas fiscales para impulsar el gasto } \\
\text { en la salud, subsidios salariales para retención de empleo. }\end{array}$ \\
\hline Italia & $\begin{array}{l}\text { Desigualdad en el mercado laboral. Trabajo desde } \\
\text { casa. }\end{array}$ & $\begin{array}{l}\text { Políticas destinadas a aliviar la desigualdad y medidas de } \\
\text { apoyo a los ingresos. }\end{array}$ \\
\hline
\end{tabular}




\begin{tabular}{cll}
\hline Países & Principales problemáticas & \multicolumn{1}{c}{ Estrategias } \\
\hline \multirow{3}{*}{ Malasia } & Lagunas económicas en las empresas. & $\begin{array}{l}\text { Crear un modelo para predecir los posible brotes y generar } \\
\text { estrategias para minimizar los efectos económicos en las } \\
\end{array}$ \\
& MiPymes. \\
\hline
\end{tabular}

Fuente: elaboración propia por parte de los autores.

El estudio presenta en la tabla 2 una serie de problemáticas que han surgido en las empresas a partir de la crisis económica a nivel mundial. Entre los principales inconvenientes se encuentra de manera común la crisis económica producto de la COVID-19y que provocó cierres de establecimientos comerciales, despidos de empleados en las empresas, afectaciones mentales de las personas y motivación para el suicidio dada la dificultad social. A esto se suman los problemas en los centros de salud, la disminución de los recursos para atender esta situación y el aumento de políticas fiscales para acceder a recursos económicos (Ivbijaro et al., 2021; Laborda et al., 2021; Hu and Qian, 2021).
El estudio también sugiere algunas estrategias para afrontar las problemáticas entre las que destacan la promoción de programas de salud mental para las familias afectadas, subsidios de desempleo para las familias que han tenido pérdidas laborales, adopción de políticas fiscales para disminuir la tasa impositiva de las empresas y espacios dinámicos para la seguridad de los consumidores de productos y servicios (Paul et al., 2021; Kutsar and Kurvet-Käosaar, 2021).

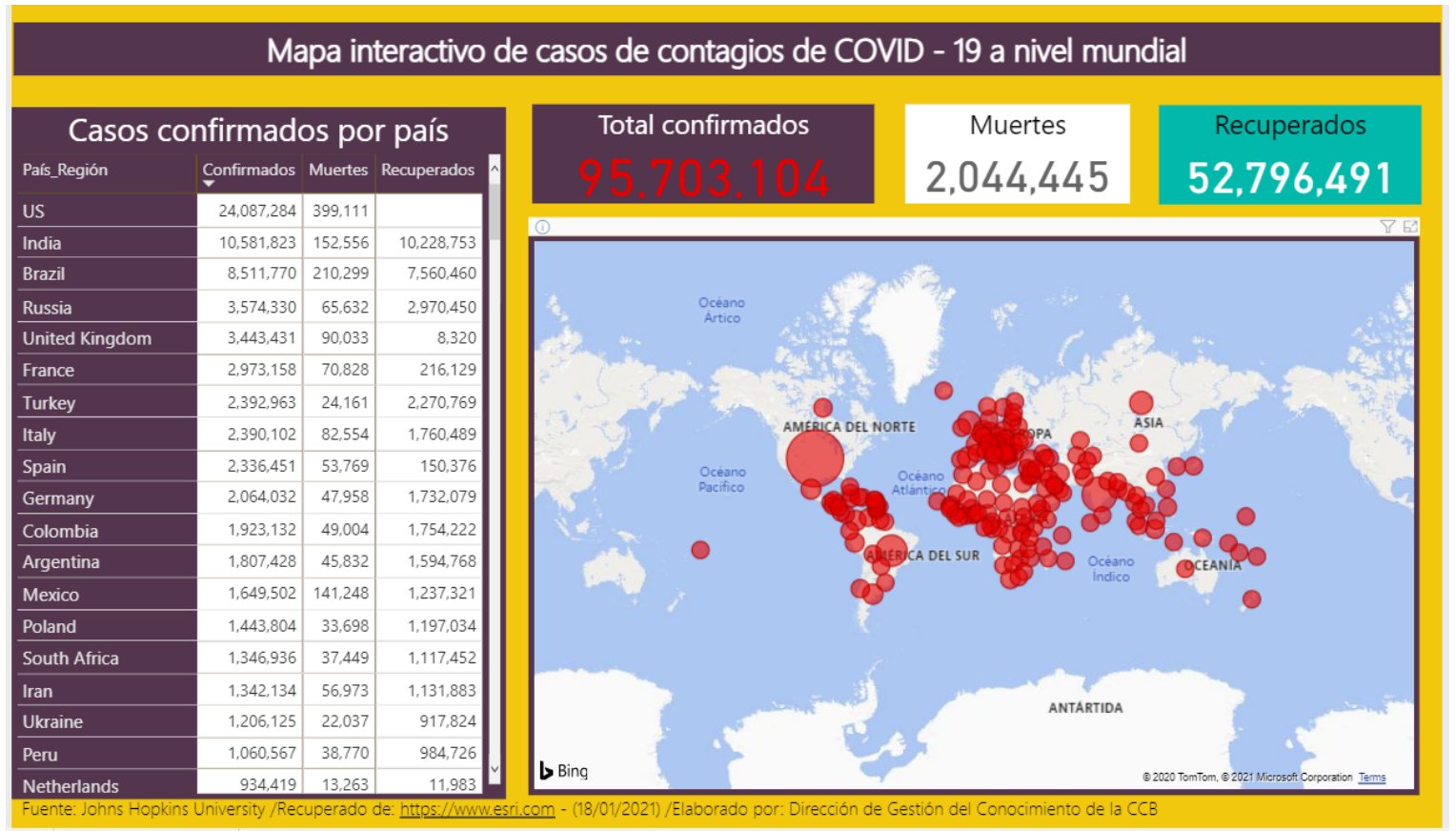

Figura 2. Casos de contagio de COVID-19 a nivel mundial para 2020. Fuente: Salud con lupa (2020). 
La figura 2 muestra las cifras de salud y contagio que han provocado que las medidas de distanciamiento social y confinamiento sean más fuertes en algunos países (Trotter, 2021). Una de las estadísticas mundiales establecidas por el Johns Hopkins University reflejó un total de 95'703104 casos de contagios y alrededor de 2'044445 muertes para 2020. En la figura 3 se puede observar a los países con mayor número de afectados por la COVID-19 en 2020 y que ubica a Colombia en el puesto 11 por encima de países como Argentina, México y Perú.

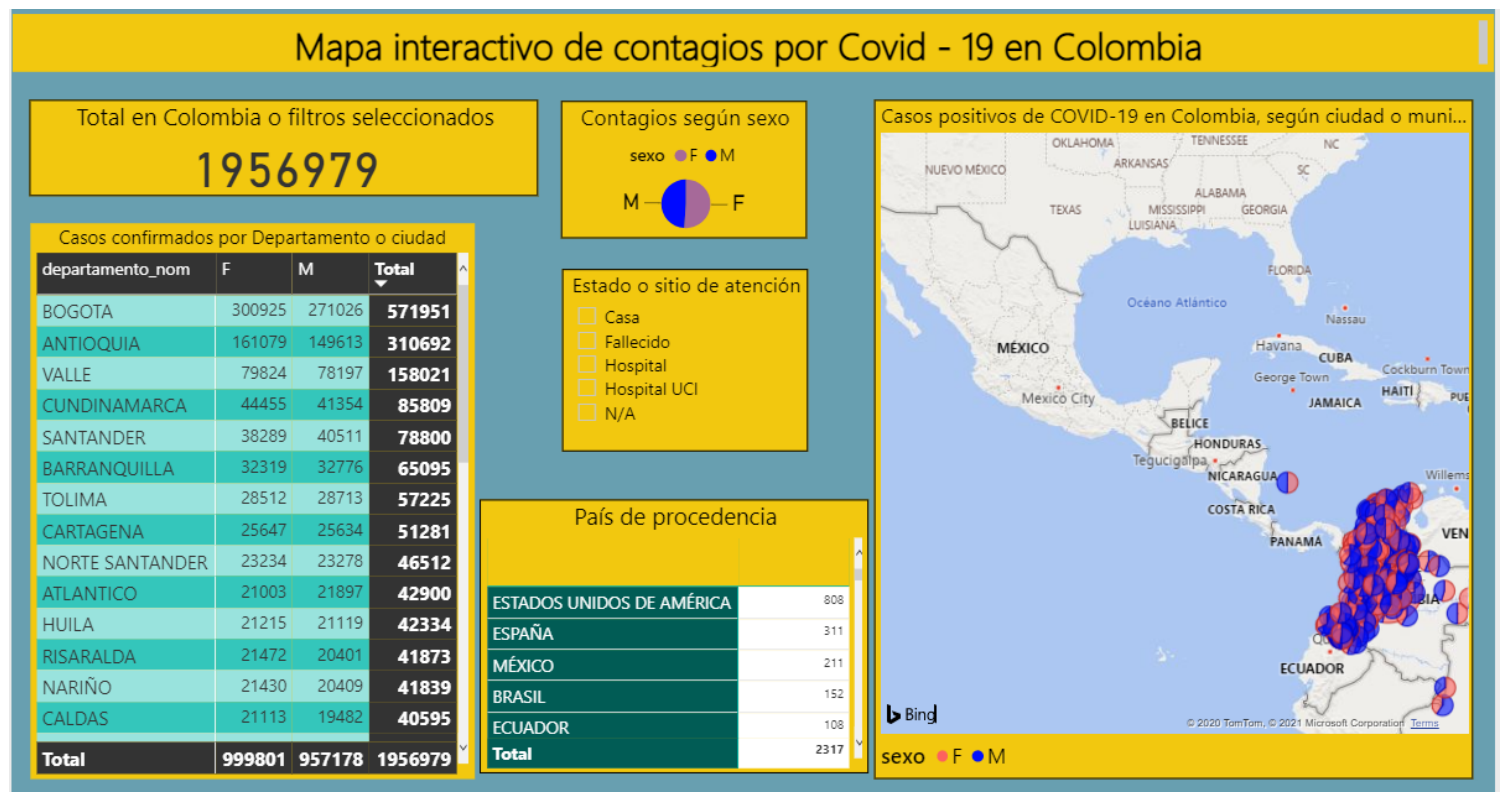

Figura 3. Contagios por COVID-19 en Colombia para 2020. Fuente: Cámara de Comercio de Bogotá (2020).

De acuerdo a la figura 3, en un análisis del territorio colombiano, se pueden percibir las zonas con mayor afectación en el país entre las que destacan Bogotá, Antioquia, Valle, Cundinamarca, con un promedio superior a las 85809 personas confirmadas frente al contagio. La figura también muestra la procedencia de los mayores casos en países como Estados Unidos, España, México, Brasil yEcuador. Este escenario tiene una gran relación con las medidas de distanciamiento social. Las regiones más afectadas por la COVID-19 han adoptado restricciones más rígidas en la movilidad y en las actividades económicas. Sin duda, estas medidas están totalmente relacionadas con la realidad que viven estos países a nivel mundial (Yarovaya et al., 2021) respecto al efecto de las medidas sociales y al efecto económico en las empresas.
Con el fin de mitigar los efectos económicos de los sectores empresariales, el presidente en su función legislativa extraordinaria- ha expedido una serie de decretos con fuerza de ley para beneficiarlos a través de subsidios de nómina, primas, devoluciones de impuestos y descuentos en pago de obligaciones fiscales del año anterior. Aunque la realidad que se presenta en el contexto colombiano es similar a la descrita a nivel mundial por Makin y Layton (2021) en la que los beneficios adoptados fueron insuficientes, principalmente para las MiPymes que no lograron cumplir con los requisitos legales para acceder a dichas ayudas.

Respecto a ello es preciso establecer que las políticas gubernamentales son deficientes y no tienen en cuenta las realidades sociales. La población más afectada en los cierres de establecimientos fueron las MiPymes debido a la 
carencia de formalidad; además de las limitaciones para acceder a fuentes de financiación según lo establecido por el gobierno como alternativa, pues les fueron negadas precisamente por su carácter informal. Por el contrario, los efectos positivos de los beneficios económicos y las medidas de los gobernantes fueron aprovechadas por las grandes empresas.

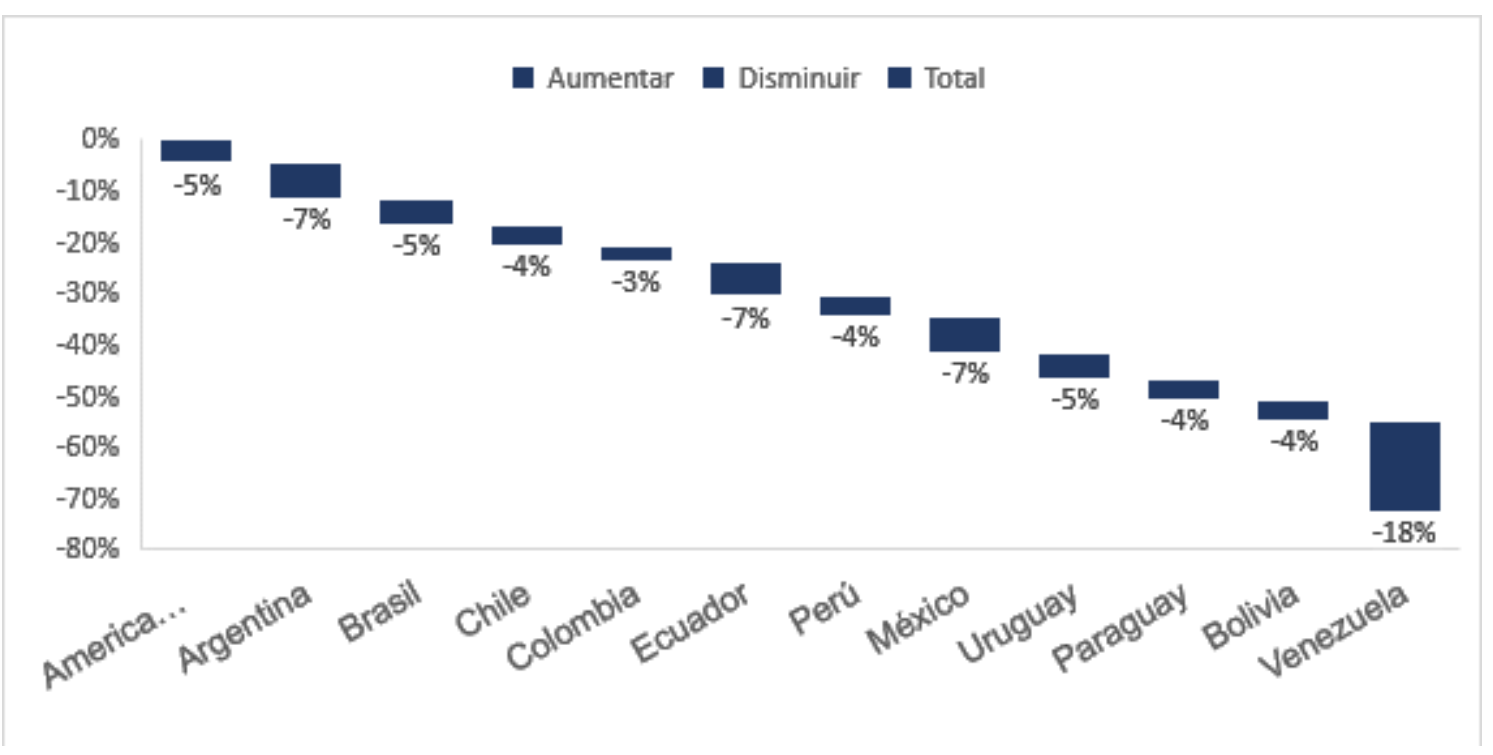

Figura 4. Proyección de crecimiento económico en 2020. Fuente: elaboración propia por parte de los autores.

Otro aspecto destacableque afecta directamente al capital humano en las organizaciones es la proyección de crecimiento. Obviamente la disminución económica, así como el funcionamiento de la actividad empresarial, afecta la calidad del empleo y aumenta la cancelación de los contratos laborales. En la figura se puede observar la tasa de crecimiento proyectada para 2020: América Latina presenta una disminución del $5 \%$; de igual manera Venezuela presenta el mayor porcentaje con el $18 \%$; mientras que Colombia presenta un $3 \%$ por debajo de países como Argentina, Brasil, Chile, Ecuador, Uruguay, Paraguay y Bolivia. Como se puede visualizar en cuanto a casos positivos Colombia llegó a estar por encima del número de contagios de estos países. Países, cuyo manejo por regiones fue más favorable para evitar efectos en la economía nacional. 


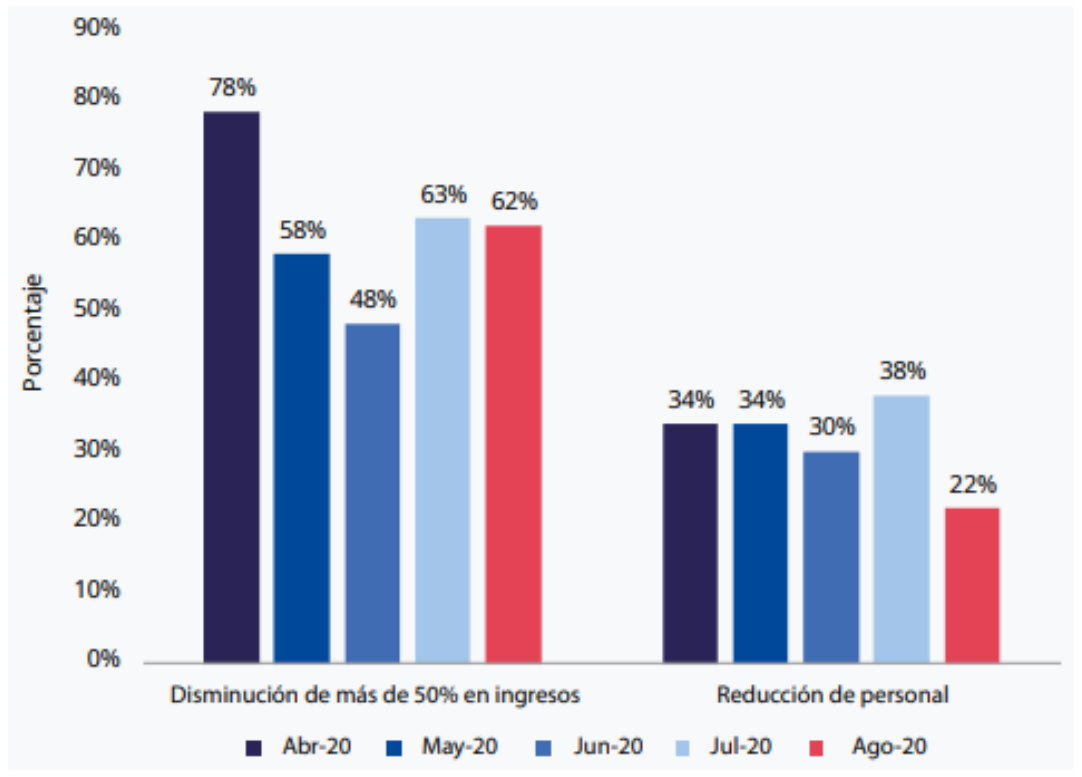

Figura 5. Disminución de ingresos y reducción de personal. Fuente: elaboración propia por parte de los autores.

La figura 5 expone una serie de aspectos que han afectado al capital humano, mostrando relación con la situación que se presenta a nivel global. La pérdida de empleo es uno de los indicadores sociales más fuertes de la economía en el mundo porque, además de afectar a los ingresos familiares y a la capacidad de sostenimiento, también logra irrumpir aspectos tales como el acceso a la educación, salud y estabilidad social del hogar. La figura presenta resultados obtenidos de la región de Bogotá en Colombia, el territorio más golpeado por la pandemia del COVID-19; en donde se ha visto una pérdida de 370 a 550 mil empleos. Una cifra demasiada alta que afecta a miles de familias que se quedaron sin el sustento mínimo para vivir.

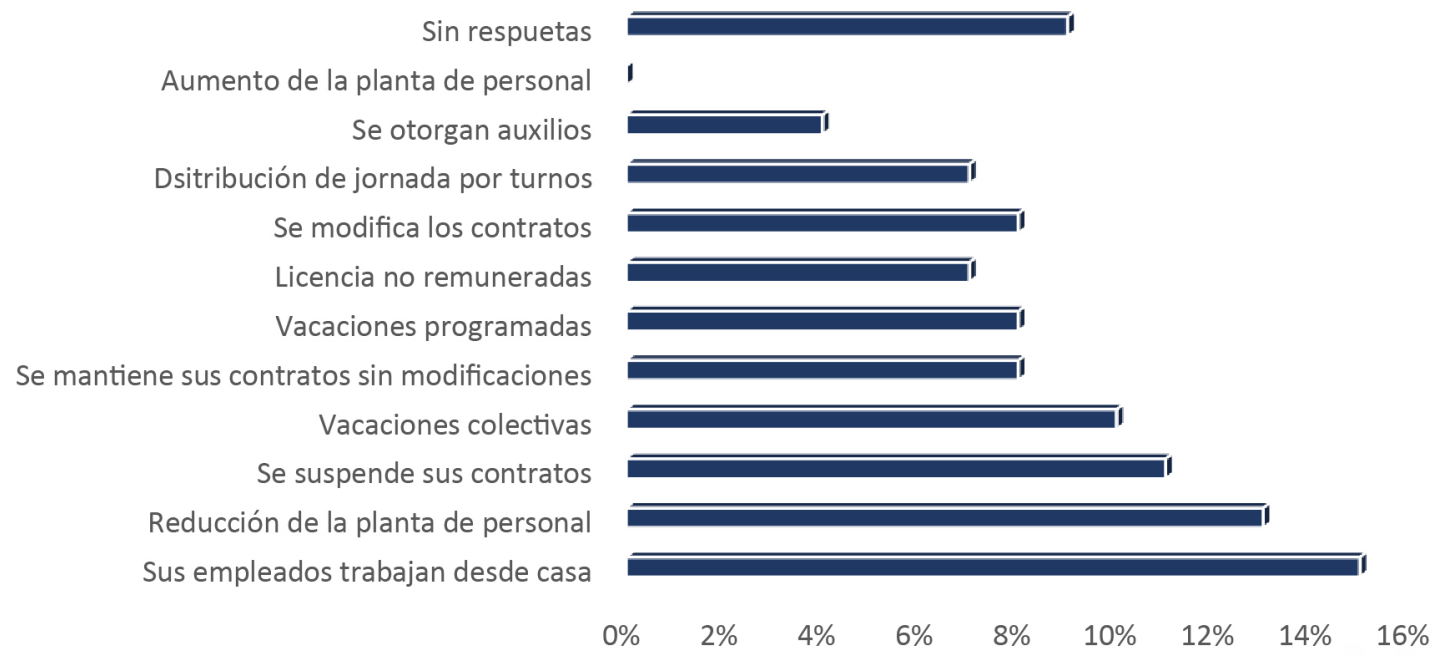

Figura 6. Medidas implementadas en las empresas para el capital humano ante la situación provocada por la COVID-19. Fuente: elaboración propia por parte de los autores. 
La figura 6 plantea las medidas adoptadas por las distintas empresas colombianas respecto a su capital humano, destacando principalmente entre las estrategias empresariales: el trabajo en casa, en un $15 \%$; la reducción de personal, producto del cese de actividades y los niveles de servicio o producción, en un $13 \%$; la suspensión de contratos hasta que las actividades regresen a la normalidad, en un $11 \%$. Asimismo, otras estrategias que se implementaron para mantener su capital humano fueron las modificaciones contractuales y la congelación de salarios.

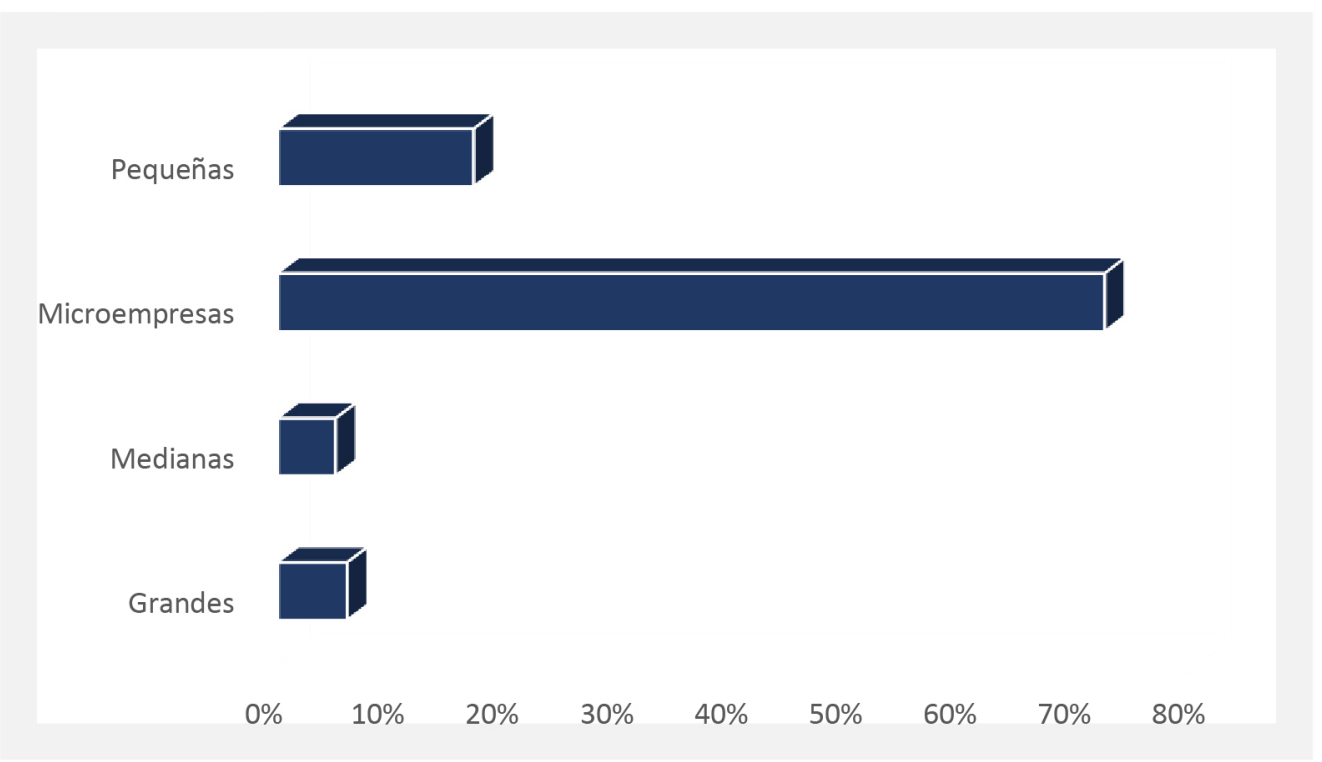

Figura 7. Empresas más afectadas por la crisis de la COVID-19. Fuente: elaboración propia por parte de los autores.

La figura 7 muestra que el sector empresarial más afectado por la crisis económica y social fue el de las MiPymes con un $72 \%$ de las afectaciones. Dato que concuerda con lo mencionado por $\mathrm{Lu}$ et al. (2020), quienes establecen que los tejidos empresariales están conformados principalmente por las microempresas y que a la vez es el sector económico más vulnerable ante la crisis. Este planteamiento concuerda con la situación de Colombia, la cual presenta una cifra alta en el cese de actividades del sector microempresarial debido a que tienen un bajo nivel de capital y por consiguiente sus flujos económicos son débiles para sostenerse sin el desarrollo de su actividad. Además es preciso mencionar que las políticas adoptadas por los gobiernos locales y nacionales en subsidios de la nómina y beneficios fiscales no favorecieron a este segmento del tejido empresarial, ya que destaca su informalidad y la imposibilidad de cumplir con los requisitos y requerimientos planteados por las entidaes financeiras para acceder a los subsidios y subvenciones. 


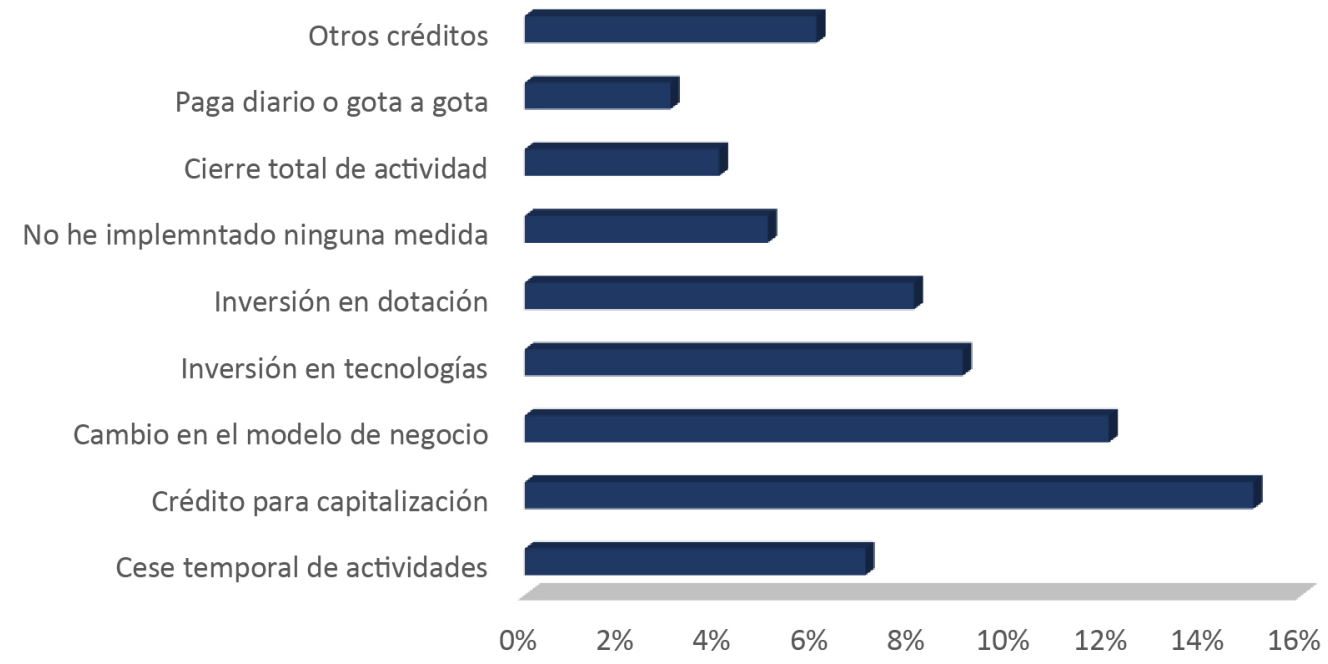

Figura 8. Medidas adoptadas por las empresas debido a la COVID-19. Fuente: elaboración propia por parte de los autores.

La figura 8 presenta las medidas adoptadas por las empresas frente a la COVID-19, en especial el cese temporal de actividades en un $70 \%$ del sector comercio. Escenario similar al contexto latinoamericano establecido por la CEPAL, la cual señala que la mayor parte de las empresas han adoptado el cierre temporal - y en algunos casos el cierre definitivo de sus actividades- por la incapacidad económica de pagar sus gastos fijos de nóminas y gastos operacionales.

Respecto a la decisión de capitalización adoptada, el indicador muestra un $15 \%$ con un resultado muy bajo debido a factores tales como los requisitos formales que limitan el acceso a la financiación. Por su puesto, este indicador esta correlacionado con el tipo de empresas más afectadas. Las MiPymes, por su carácter informal y poca capacidad organizativa, se encuentran limitadas por los requisitos establecidos por el sector bancario para acceder a las líneas de crédito; lo anterior, imposibilita la obtención de recursos de capital para sostener su actividad económica.

Otro aspecto que se destaca es el de las inversiones en tecnología, es claro que las medidas de distanciamiento social y el confinamiento hicieron que los clientes optaran por las compras en línea; esto obligó a las empresas a implementar nuevos sistemas tecnológicos. Afirmación que concuerda con lo mencionado por Soelasih y Jaya
(2020), quienes describieron el uso de plataformas tecnológicas como medida urgente para mantener las ventas y suplir la necesidad de los compradores.

Este trabajo presenta una visión de las afectaciones del capital humano en las empresas, especialmente en las MiPymes que son las que han sufrido múltiples problemas a raíz de la crisis económica y social. En el mundo las estrategias de distanciamiento social, así como el confinamiento han sido las medidas de prevención más usadas para prevenir el contagio y sus resultados han sido favorables para disminuir la tasa de positivos. Aunque dichas medidas fueron desfavorables para los sectores económicos del tejido empresarial dado que vieron como sus ventas disminuían por las políticas de confinamiento, distanciamiento y limitaciones en la movilidad en los territorios.

Si bien los gobiernos nacionales han permitido la reactivación gradual de la economía bajo el cumplimiento de protocolos de bioseguridad, los índices de cierres y pérdidas de empleo siguen en crecimiento. Por su parte la apertura parcial de ciertas actividades (como, por ejemplo, el turismo y los restaurantes) han ayudado a mejorar los ingresos económicos de las MiPymes, pero también han demostrado que esas decisiones son directamente proporcionales a las tasas de multiplicación del virus. 
En todo el mundo, los gobiernos han implementado estrategias para minimizar los efectos de la emergencia social entre las que se encuentran los subsidios económicos para que los empresarios cubran los gastos de nómina. Sin embargo esta estrategia solo ha favorecido a los grandes grupos económicos puesto que las MiPymes por sus características informales no logran acceder a los beneficios y subvenciones gubernamentales.

Otras acciones de los gobiernos para mejorar los índices de cierres de establecimientos fue la apertura gradual de las actividades de los sectores primarios, los cuales son los responsables de los recursos y de la capacidad alimentaria del país. No obstante, han tenido dificultades con los procesos de transporte y circulación de productos.

\section{Conclusiones}

En relación con el impacto en el capital humano como consecuencia de la pandemia de la COVID-19 el de mayor proporción fue la pérdida de empleos debido al cierre de muchas empresas y de la recesión en la actividad económica que afectó la dinámica empresarial, la entrada de nuevos negocios y con ello la afectación a los que estaban buscando empleo. Todo esto sin contar con los altos índices de estrés para los trabajadores que mantuvieron sus puestos, así como la afectación a sus familias por la implementación del trabajo en casa.

Dentro de las limitaciones del estudio sobre el capital humano en las MiPymes a partir de la crisis económica y social del COVID-19 se tiene la revisión de textos académicos de alto impacto científico en la base de datos Scopus. No obstante, hay otros estudios publicados por organizaciones mundiales y revistas indexadas en otras bases de datos que pueden ofrecer información relevante para entender las problemáticas relacionadas con el capital humano en las empresas. Se recomienda, entonces, para próximos estudios, usar múltiples bases de datos e incorporar los reportes de organismos mundiales que hagan posible realizar un mayor aporte.

\section{Referencias}

Ahn, S. and Norwood, F. (2021). Measuring Food Insecurity during the COVID-19 Pandemic of Spring 2020.Applied Economic Perspectives and Policy, 43(1), 162-168.

Amuda, Y. (2020). Impact of Coronavirus on Small and Medium Enterprises (SMEs): Towards Post-COVID-19 Economic Recovery in Nigeria. Academy of Strategic Management Journal, 19(6), 1-11.

Bartik, A. et al. (2020). The impact of COVID-19 on small business outcomes and expectations. Proceedings of the National Academy of Sciences of the United States of America, 117(30), 1765617666.

Bikse, V. et al. (2021). The Development of Digital Transformation and Relevant Competencies for Employees in the Context of the Impact of the COVID-19 Pandemic in Latvia. Sustainability, 13(16), 9233.

Burhan, M. et al. (2021). Crisis management in the hospitality sector SMEs in Pakistan during COVID-19. International Journal of Hospitality Management, 98, 103037.

Cámara de Comercio de Bogotá. (2020). Mapas interactivos-Colombia. Recuperado de https:// www.ccb.org.co/observatorio/Analisis-Social/ Analisis-Social/Salud-y-Covid-19/Mapasinteractivos-Colombia.

CEPAL. (2020). Sectores y empresas frente al COVID-19: emergencia y reactivación. Santiago de Chile, Chile: CEPAL.

David, K. et al. (2021). Increased Risk of Death Triggered by Domestic Violence, Hunger, Suicide, Exhausted Health System during COVID-19 Pandemic: Why, How and Solutions. Frontiers in Sociology, 6, 1-6.

Davila, F. etal.(2021). COVID-19 and food systems in Pacific Island Countries, Papua New Guinea, and Timor-Leste: Opportunities for actions towards the sustainable developmentgoals. Agricultural Systems, 191, 103137. 
Eggers, F. (2020). Masters of disasters? Challenges and opportunities for SMEs in times of crisis. Journal of Business Research, 116, 199-208.

Ejiogu, A., Okechukwu, O. and Ejiogu, C. (2020). Nigerian budgetary response to the COVID-19 pandemic and its shrinking fiscal space: financial sustainability, employment, social inequality and business implications. Journal of Public Budgeting, Accounting and Financial Management, 32(5), 919-928.

Fuentes-Doria, D. etal.(2020). Prácticas degestión sostenible y políticas gubernamentales: una mirada de las acciones mundiales. En Canabal, J. et al. (Eds.), Business Intelligence Big Data y contabilidad tridimensional: un reto prospectivo a la competitividad de las organizaciones (pp. 106-119). Montería, Colombia: Universidad del Sinú.

Fuentes-Doria, D., Toscano-Hernández, A. and Sepulveda, R. (2019). Enterprise Sustainability: Literature Review in the Context of SMEs Worldwide. Opción, 35(25), 1526-1558.

Grashuis, J. (2021). Self-employment duration during the COVID-19 pandemic: A competing risk analysis. Journal of Business Venturing Insights, 15, e00241.

$\mathrm{Hu}$, Y. and Qian, Y. (2021). COVID-19, Interhousehold Contact and Mental Well-Being Among Older Adults in the US and the UK. Frontiers in Sociology, 6, 1-15.

Iacus, S. et al. (2020). Estimating and projecting air passenger traffic during the COVID-19 coronavirus outbreak and its socio-economic impact. Safety Science, 129, 104791.

Islam, R. (2020). Assessing the challenges in online class during the coronavirus (COVID-19) pandemic in Bangladesh. Academy of Strategic Management Journal, 19(1), 1-8.

Ivbijaro, G. et al. (2021). Suicide prevention and COVID-19. Asia-Pacific Psychiatry, 13(3), 1-12.

Jílková, P. (2021). Sustainable Corporate Strategy: The Role of Human Capital in the Time of COVID-19 Crisis. TEM Journal, 10(2), 699-706.
Kim, A. et al. (2021). COVID-19 and the decline in Asian American employment. Research in Social Stratification and Mobility, 71, 100563.

Konečný, V., Brídziková, M. and Senko, Š. (2021). Impact of COVID-19 and anti-pandemic measures on the sustainability of demand in suburban bus transport. The case of the Slovak Republic. Sustainability, 13(9), 4967.

Korsgaard, S. et al. (2020). COVID-19 and the importance of space in entrepreneurship research and policy. International Small Business Journal: Researching Entrepreneurship, 38(8), 697-710.

Kutsar, D. and Kurvet-Käosaar, L. (2021). The Impact of the COVID-19 Pandemic on Families: Young People's Experiences in Estonia. Frontiers in Sociology, 6, 1-12.

Kyung, A. and Whitney, S. (2020). A study on the financial and entrepreneurial risks of small business owners amidst COVID-19. En International IOT, Electronics and Mechatronics Conference (IEMTRONICS), Toronto, Canada.

Laborda, J. et al. (2021). Is there life beyond the Spanish government's aid to furloughed employees by COVID-19? PLOS ONE, 16(6), 1-21.

Larue, B. (2020). Labor issues and COVID-19. Canadian Journal of Agricultural Economics, 68(2), 231-237.

$\mathrm{Lu}, \mathrm{Y}$. et al. (2020). The perceived impact of the COVID-19 epidemic: evidence from a sample of 4807 SMEs in Sichuan Province, China. Environmental Hazards, 19(4), 323-340.

Makin, A.J. and Layton, A. (2021). The global fiscal response to COVID-19: Risks and repercussions. Economic Analysis and Policy, 69, 340-349.

Mamun, M., Bhuiyan, A.K.M.I. and Manzar, M. (2020). The first COVID-19 infanticide-suicide case: Financial crisis and fear of COVID-19 infection are the causative factors. Asian Journal of Psychiatry, 54, 102365.

Mirza, N. etal. (2020). The impact of human capital efficiency on Latin American mutual funds 
during COVID-19 outbreak. Swiss Journal of Economics and Statistics, 156(16), 2-7.

Nande, A. et al. (2021). Dynamics of COVID-19 under social distancing measures are driven by transmission network structure. PLoS Computational Biology, 17(2), 1-26.

Nga,J.L.H., Ramlan, W.and Naim, S. (2021).COVID-19 pandemic and unemployment in Malaysia: A case study from Sabah. Cosmopolitan Civil Societies, 13(2), 73-90.

Nwosimiri, O. (2021). COVID-19 and Job Losses: Should Affirmative Action and Preferential Hiring still be Applicable in South Africa? Filosofia Theoretica: Journal of African Philosophy, Culture and Religions, 10(1), 1-18.

Nwosu, C. and Oyenubi, A. (2021). Incomerelated health inequalities associated with the coronavirus pandemic in South Africa: A decomposition analysis. International Journal for Equity in Health, 20(1), 1-12.

Palma, J., Araos, C. and Moore, E. (2021). Household Coping Strategies During the COVID-19 Pandemic in Chile. Frontiers in Sociology, 6, 1-14.

Paul, R. et al. (2021). The Association of Social Determinants of Health with COVID-19 Mortality in Rural and Urban Counties. Journal of Rural Health, 37(2), 278-286.

Pinilla, J. et al. (2021). The Economic Impact of the SARS-COV-2 (COVID-19) Pandemic. Research in Transportation Economics, 2, 101090.

Restrepo, B., Rabbitt, M. and Gregory, C. (2021). The Effect of Unemployment on Food Spending and Adequacy: Evidence from Coronavirus-Induced Firm Closures. Applied Economic Perspectives and Policy, 43(1), 185-204.

Rodríguez, R., Díaz, J. y Niebles, L. (2018). Gestión humana y su estado actual en las pymes de Montería. En Castro, L., Niebles, W. y De la Hoz, J.D. (Comps.), Desarrollo organizacional: una mirada a micro, pequeñaymediana empresa (pp. 39-63). Barranquilla, Colombia: Corporación Universitaria Latinoamericana.
Salud con lupa. (2020). Mapa interactivo: la pandemia de coronavirus en tiempo real. Recuperado de https://saludconlupa.com/ noticias/mapa-interactivo-la-epidemia-decoronavirus-en-tiempo-real/.

Schmitz, L. et al. (2021). The impact of late-career job loss and genetic risk on body mass index: Evidence from variance polygenic scores. Scientific Reports, 11(1), 1-15.

Shammi, M. et al. (2020). Strategic assessment of COVID-19 pandemic in Bangladesh: comparative lockdown scenario analysis, public perception, and managementfor sustainability. Environment, Development and Sustainability, $23,6148-6191$.

Soelasih, Y. and Jaya, A. (2020). Strategies to maintain aviation consumers during the COVID-19 pandemic in Indonesia. Academy of Strategic Management Journal, 19(1), 1-13.

Song, H., Yang, Y. and Tao, Z. (2020). How different types of financial service providers support small- and medium- enterprises under the impact of COVID-19 pandemic: from the perspective of expectancy theory. Frontiers of Business Research in China, 14(1), 1-27.

Svabova, L. et al. (2021). Evaluation of the impacts of the COVID-19 pandemic on the development of the unemployment rate in Slovakia: Counterfactual before-after comparison. Equilibrium. Quarterly Journal of Economics and Economic Policy, 16(2), 261-284.

Toscano-Hernández, A. et al. (2020). Análisis y evaluación de la sostenibilidad empresarial: herramientas de apoyo a la toma de decisiones y la medición del rendimiento en pymes. En Canabal, J. et al. (Eds.), Business Intelligence Big Data y contabilidad tridimensional: un reto prospectivo a la competitividad de las organizaciones (pp. 225-233). Montería, Colombia: Universidad del Sinú.

Toscano-Hernández, A., Fuentes-Doria, D. y Fajardo-Pereira, M. (2019). Sostenibilidad con enfoque en la educación ambiental: redes de colaboración y científica mundial. Panorama Económico, 27(1), 60-84. 
Trotter, S. (2021). Ways of Being Together During the COVID-19 Pandemic: Support Bubbles and the Legal Construction of Relationships. Frontiers in Sociology, 6, 1-10.

Villalobos, G. et al. (2021). Desempleo juvenil: contribuciones para su disminución desde el emprendimiento. Revista Venezolana de Gerencia, 26(95), 758-775.

Wahyono, H. et al. (2021). Irrationality and economic morality of SMEs' behavior during the COVID-19 pandemic: lesson from Indonesia. Heliyon, 7(7), e07400.

Yarovaya, L. etal. (2021). Human Capital efficiency and equity funds' performance during the COVID-19 pandemic. International Review of Economics and Finance, 71, 584-591.

Zimon, G. and Dankiewicz, R. (2020). Trade credit management strategies in SMEs and the COVID-19 pandemic-a case of Poland. Sustainability, 12(15), 6114. 